The Simplex Method for Quadratic Programming Author(s): Philip Wolfe

Source: Econometrica, Vol. 27, No. 3, (Jul., 1959), pp. 382-398

Published by: The Econometric Society

Stable URL: http://www.jstor.org/stable/1909468

Accessed: $22 / 07 / 2008$ 04:45

Your use of the JSTOR archive indicates your acceptance of JSTOR's Terms and Conditions of Use, available at http://www.jstor.org/page/info/about/policies/terms.jsp. JSTOR's Terms and Conditions of Use provides, in part, that unless you have obtained prior permission, you may not download an entire issue of a journal or multiple copies of articles, and you may use content in the JSTOR archive only for your personal, non-commercial use.

Please contact the publisher regarding any further use of this work. Publisher contact information may be obtained at http://www.jstor.org/action/showPublisher?publisherCode=econosoc.

Each copy of any part of a JSTOR transmission must contain the same copyright notice that appears on the screen or printed page of such transmission.

JSTOR is a not-for-profit organization founded in 1995 to build trusted digital archives for scholarship. We work with the scholarly community to preserve their work and the materials they rely upon, and to build a common research platform that promotes the discovery and use of these resources. For more information about JSTOR, please contact support@jstor.org. 


\title{
THE SIMPLEX METHOD FOR QUADRATIC PROGRAMMING
}

\author{
By Philip Wolfe
}

\begin{abstract}
A computational procedure is given for finding the minimum of a quadratic function of variables subject to linear inequality constraints. The procedure is analogous to the Simplex Method for linear programming, being based on the Barankin-Dorfman procedure for this problem.
\end{abstract}

\section{INTRODUCTION}

IN THIS PAPER, by "quadratic programming" we shall understand the problem of determining values of several real variables, subject to linear inequality constraints, which yield the extreme value of a quadratic function. Besides being a step on the way toward solution of the elaborate nonlinear programming problems which economic models often present, a usable computational procedure for quadratic programming can be applied to a number of problems of interest in themselves:

Regression. To find the best least-squares fit to given data, where certain parameters are known a priori to satisfy inequality constraints (e.g., being nonnegative).

Efficient production. Maximization of profit, assuming linear production functions and linearly varying marginal costs.

"Portefolio" problem. ${ }^{1}$ To find a combination of random variables having given expectation and minimum variance.

Convex programming. To find the minimum of a general convex function under linear constraints using a quadratic approximation.

Let the variables of the problem constitute the $n$-vector $x=\left(x_{1}, \ldots, x_{n}\right)^{\prime}$ (' will denote transposition; we take $x$ to be a column vector, that is, an $n$ by 1 matrix). Letting $A$ be an $m$ by $n$ matrix, and $b$ an $m$ by 1 , we shall express the linear constraints of the problem by

$$
x \geq 0, \quad A x=b,
$$

that is, $\quad x_{j} \geq 0(j=1, \ldots, n), \quad \sum_{j=1}^{n} a_{i j} x_{j}=b_{i}(i=1, \ldots, m)$.

We recall that any combination of linear equation and inequality constraints can be written in the form (1) if additional variables are allowed into the problem.

Let $p$ be a 1 by $n$ matrix, and $C$ be an $n$ by $n$ symmetric matrix. We shall write the objective function, the (partly) quadratic function to be extremized subject to (1), as

$$
f(\lambda, x)=\lambda p x+1 / 2 x^{\prime} C x,
$$

1 Jue to Markowitz [6]. 
or

$$
f(\lambda, x)=\lambda \Sigma_{j} p_{j} x_{j}+1 / 2 \Sigma_{j, k} x_{j} C_{j k} x_{k},
$$

where $\lambda$, a single nonnegative real parameter, can be chosen as convenient. The problem can now be stated as:

The quadratic problem for $\lambda \geq 0$ :

$$
\text { Minimize } f(\lambda, x)=\lambda p x+1 / 2 x^{\prime} C x \quad \text { subject to } x \geq 0, A x=b \text {. }
$$

An important restriction must be placed on the quadratic part, $C$, of the objective function in order to ensure the success of the computational method: the function $f$ must be convex, that is, $C$ must be positive semidefinite. This condition-apparently essential, in the present state of the art, to all nonlinear programming schemes-ensures that any local minimum encountered in the problem will be the global solution desired. Algebraically, the assertion of positive semidefiniteness for $C$ is that

$$
x^{\prime} C x \geq 0 \text { for all } x .
$$

In economic problems, it is the ascription of nonincreasing returns to scale for all activities, since the marginal cost of changing from the program $x$ to the program $x+\Delta x$ is given by

$$
\frac{d}{d t} f(\lambda, x+t \triangle x)=p \triangle x+x C \triangle x+t \triangle x^{\prime} C \triangle x
$$

which will be a nondecreasing function of $t$. We shall assume it from now on. A more detailed discussion of the role of this property in quadratic programming is given in [4].

A number of proposals for the computational solution of quadratic programming problems have been made in the last two years; those which seem suited to high-speed digital computers are given in References [1]--[6] below. Barankin and Dorfman [1] first pointed out the linear formulation of the quadratic problem, inspiring the present approach; our Section 2 is taken, with changed notation, from Sections 1 and 3 of [1].

The principal respect in which the present method differs from these is in using only the computational mechanisms of the Simplex Method for linear programming problems. It is therefore a simple matter to convert a computing machine code for linear programming into one for quadratic programming; the SHARE linear programming code for the IBM 704 requires modification in eleven instructions for this purpose.

In the sequel this method is developed in two forms, "short" and "long." The computations of the long form are like those of the short, but are aimed at solving the quadratic problem (3) for all $\lambda \geq 0$ and at the same time avoiding certain restrictions on the use of the short form. The table below summarizes the conditions for use of these methods. The estimate of the number of Simplex Method changes-of-basis needed to solve the problem is based on experience like that described in Section 6 . 
Solution of (3) by:

Conditions

Solution obtained for

Size of equivalent linear program

Estimated number of basis changes for solution
Short Form

Long Form

\begin{tabular}{c|c}
\hline $\begin{array}{c}\text { Either } \lambda=0 \text { or } C \\
\text { positive definite }\end{array}$ & $C$ positive semidefinite \\
\hline fixed $\lambda$ & all $\lambda \geqslant 0$ \\
\hline $\begin{array}{c}\text { up to } m+n \text { equations, } \\
m+3 n \text { variables }\end{array}$ & $\begin{array}{c}\text { up to } m+n \text { equations, } \\
m+3 n+1 \text { variables }\end{array}$ \\
\hline $2(m+n)$ & $4(m+n)$ \\
\hline
\end{tabular}

\section{PRELIMINARIES}

Since we are interested, in part, in the solution of the quadratic problem for all $\lambda \geq 0$, let us define

$$
\text { for } \lambda \geq 0: F(\lambda)=\operatorname{Min}\left\{\lambda p x+1 / 2 x^{\prime} C x: x \geq 0, A x=b\right\} \text {. }
$$

Quite a bit of information about $F(\lambda)$ can be obtained without calculating it. Throughout it will be assumed, of course, that there exist feasible $x$, i.e., $x \geq 0$ such that $A x=b$. Nevertheless, we may have $F(\lambda)=-\infty$ for some (and hence all) $\lambda>0$.

First, an important feature of the positive semidefiniteness of $C$ is given by

Lemma 1. $x^{\prime} C x=0$ implies $C x=0$.

Proof. For any $n$-vector $y$ we have

$0 \leq(y+t x)^{\prime} C(y+t x)=y^{\prime} C y+2 t y^{\prime} C x$ for any number $t$, whence $y^{\prime} C x=0$; $C x=0$ follows at once.

This leads to

LEMMA 2. For any $\lambda \geq 0$, the "solution set" of all feasible $x$ such that $f(\lambda, x)=$ $F(\lambda)$ is the intersection of a linear manifold with the constraint set, and $p x$ is constant on this set for $\lambda>0$.

Proof. Let feasible points $x, y$ be given such that $f(\lambda, x)=f(\lambda, y)=F(\lambda)$. Letting $w=y-x$, for any $0 \leq t \leq 1$ the point $x+t w$ belongs to the constraint set; since $f$ is convex, and $f(\lambda, x+t w)$ is minimal for $t=0$ and $t=1$, we have $f(\lambda, x+t w)=f(\lambda, x)$ for all $0 \leq t \leq 1$, or $\lambda p(x+t w)+1 / 2(x+t w)^{\prime}$ $C(x+t w)=\lambda p x+1 / 2 x^{\prime} C x$, which simplifies to $\left(\lambda p w+x^{\prime} C w\right) t+1 / 2$ $w^{\prime} C w t^{2}=0[0 \leq t \leq 1]$. Thus $w^{\prime} C w=0$, whence by Lemma 1

$$
\left\{\begin{array}{l}
C w=0, \text { and hence } \\
p_{w}=0 .
\end{array}\right.
$$


Conversely, it is clear that if $f(\lambda, x)=F(\lambda)$, if $w$ satisfies (5), and if $x+t w$ is feasible, then $f(\lambda, x+t w)=F(\lambda)$, so that the complete solution set for $\lambda$ given is the intersection of the constraint set with the linear manifold $\{x+t w\}$. Equation (5) yields finally $p x=p y$ for any two solutions.

If now for any $\lambda>0$ we choose a feasible solution $x_{\lambda}$ such that $f\left(\lambda, x_{\lambda}\right)=$ $F(\lambda)$, then by Lemma 2 the value $p x_{\lambda}$ is independent of the choice of $x_{\lambda}$.

THEOREM 1. For $\lambda \geq 0, F(\lambda)$ is a concave function; $p x_{\lambda}$ is monotone nonincreasing; and $x_{\lambda}$ is a solution $y$ of the problem:

$$
\operatorname{Min}\left\{y^{\prime} C y: y \geq 0, A y=b, p y \leq p x_{\lambda}\right\} \text {. }
$$

Proof. Since $f(\lambda, x)$ is linear in $\lambda$, the function $F(\lambda)$ is the iniimum of a family of linear functions, and hence concave.

The trend of $p x_{\lambda}$ is an instance of a quite general fact. Take any $\lambda$ and $\mu$. Since $x_{\lambda}$ minimizes $f(\lambda, x)$, we have

$$
\lambda p x_{\lambda}+1 / 2 x_{\lambda}^{\prime} C x_{\lambda} \leq \lambda p x_{\mu}+1 / 2 x_{\mu}^{\prime} C x_{\mu} ;
$$

and since $x_{\mu}$ minimizes $f(\mu, x)$, we have

$$
\mu p x_{\mu}+1 / 2 x_{\mu}^{\prime} C x_{\mu} \leq \mu p x_{\lambda}+1 / 2 x_{\lambda}^{\prime} C x_{\lambda} .
$$

Adding these inequalities and rearranging, we get

$$
(\mu-\lambda) p x_{\mu} \leq(\mu-\lambda) p x_{\lambda},
$$

which yields $p x_{\mu} \leq p x_{\lambda}$ for $\mu>\lambda$.

Finally, since $x_{\lambda}$ does minimize $\lambda p x+1 / 2 x^{\prime} C x$, any $y$ such that $y^{\prime} C y<$ $x_{\lambda}^{\prime} C x_{\lambda}$ will give $p y>p x_{\lambda}$, which proves the last statement.

The next theorem characterizes $x_{\lambda}$ in such a way that we will be able to compute it. Only the sufficiency of this condition for the minimization of $f(\lambda, x)$ is needed, since its necessity will follow when we have established that results of the computational scheme of the next section meet this condition if the minimum exists.

THEOREM 2. If $x \geq 0, A x=b$, and there exist $v \geq 0$ ( $v$ is $n$ by 1 ) and $u$ ( $u$ is $m$ by 1 ) such that

$$
v^{\prime} x=0
$$

and

$$
C x-v+A^{\prime} u+\lambda p^{\prime}=0,
$$

then $x$ solves the problem Min $\left\{\lambda p x+1 / 2 x^{\prime} C x: x \geq 0, A x=b\right\}$. 
Proof. Let any $y \geq 0, A y=b$ be given. We shall show that $f(\lambda, y) \geq f(\lambda, x)$. From the positive semidefiniteness of $C$ we have

whence

$$
(y-x)^{\prime} C(y-x) \geq 0,
$$

or

$$
y^{\prime} C y+x^{\prime} C x \geq 2 x^{\prime} C y
$$

$$
y^{\prime} C y-x^{\prime} C x \geq 2 x^{\prime} C(y-x)
$$

so that

$f(\lambda, y)-f(\lambda, x)=\lambda p(y-x)+1 / 2 y^{\prime} C y-1 / 2 x^{\prime} C x \geq\left(\lambda p+x^{\prime} C\right)(y-x)$.

Since by (7) $\quad \lambda p+x^{\prime} C=v^{\prime}-u^{\prime} A$,

$$
\begin{gathered}
f(\lambda, y)-f(\lambda, x) \geq v^{\prime} y-v^{\prime} x-u^{\prime} A y+u^{\prime} A x=v^{\prime} y-0-u^{\prime} b+u^{\prime} b \text { (by (6) } \\
\text { and feasibility) } \left.=v^{\prime} y \geq 0 \quad \text { (since } v, y \geq 0\right) .
\end{gathered}
$$

The conditions (6) and (7) - especially as necessary, rather than sufficient, conditions-are essentially those of the "saddlepoint" theorem of Kuhn and Tucker $[\mathbf{9}]$. In the present form, the result is due to Barankin and Dorfman $[\mathbf{1}$, Section 3$]$. The theorem in fact obtains if $f(\lambda, x)$ is replaced by any convex differentiable function and $C x+\lambda p^{\prime}$ of (7) is replaced by its gradient.

The remarkable feature of the quadratic problem is the linearity of the gradient of $f(\lambda, x)$, which confines the nonlinearity of the Kuhn-Tuckner conditions to the relation (6), $v^{\prime} x=0$, which has this sort of combinatorial expression:

$$
v_{j}>0 \text { implies } x_{j}=0(j=1, \ldots, n) .
$$

In order to explore the relation of the constraints (8) to the linear relations (7), and see how they may be handled numerically, we must consider briefly the main features of the simplex Method for linear programming [7].

It is required to minimize the linear form $c x$ under the constraints $D x=e$, $x \geq 0$ ( $D$ is $p$ by $q, c$ is 1 by $q, e$ is $p$ by 1$)$. We suppose this problem is feasible, i.e., the existence of an $x$ satisfying the constraints. It is easy to show through linear dependence that there exists a feasible $x$ having no more than $p$ positive components. A collection of $p$ columns from $D$ which correspond to the nonvanishing components of a feasible $x$ is called a basis, and $x$ is called a basic solution. In the Simplex Method one works always with such bases; given any, it is shown that either (i) the associated basic solution yields the minimum value of the linear form, or (ii) another basis differing in only one column from the given basis can be found whose associated basic solution yields a smaller value for the form, or (iii) one column can be adjoined to the basis such that a sequence of feasible $x$ 's associated with these $p+1$ columns can be found on which $c x \rightarrow-\infty$. Thus a sequence of bases is generated which terminates in either a finite or infinite solution of the problem. It is con- 
venient to make an assumption of "nondegeneracy" regarding the constraints of the problem: That any feasible vector $x$ has at least $p$ positive components. A consequence of this assumption is the linear independence of the columns of any basis. It has been shown [7] that every set of constraints can be dealt with so as to be nondegenerate. In the sequel we rely on these results, assuming nondegeneracy in the few places it is necessary.

Returning to the quadratic problem, the conditions that the $n$-vector $x$ solve the quadratic problem for $\lambda \geq 0$ may be written together as (omitting for the moment $v^{\prime} x=0$ )

$$
\begin{array}{ll}
A x & =b, \\
C x-v+A^{\prime} u+p^{\prime} \lambda & =0, \\
x \geq 0, v \geq 0, &
\end{array}
$$

or in detached coefficient form as

$$
\begin{array}{|cccc}
x \geq 0 & v \geq 0 & u & \lambda \\
A & 0 & 0 & 0 \\
C & -I & A^{\prime} & p^{\prime}
\end{array}=b
$$

constituting $m+n$ equations in $2 n$ nonnegative variables and $m$ unrestricted variables ( $\lambda$ is not considered a variable). We shall be concerned below with the basic solutions of this system. Note that the $m$ columns associated with the unrestricted variable $u$ are taken to be in every basis (this technical device makes it unnecessary algebraically to eliminate the $u$ 's to bring the system to standard form), leaving only $n$ positive variables in any basic solution to go to $x$ and $v$.

Assuming for the moment the converse of Theorem 2, if the quadratic problem has a solution, then there exist $x, v, u$ satisfying (6) and (7). But (6) implies that at least $n$ components from the $2 n x$ and $v$ vanish; and this establishes the important result of Barankin and Dorfman [1] that some basic solution of (9) constitutes a solution of the quadratic problem.

Since the computational step of the Simplex Method can be used to explore basic solutions, Barankin and Dorfman have accordingly proposed use of the method, beginning with an arbitrary basic solution of (9), to reduce $v^{\prime} x$ to zero. One method which accomplishes this is given in [4], but it is more complicated, and probably slower, than the present algorithm.

Markowitz, on the other hand, has suggested a method [6] for the "portfolio" problem (equivalent to solving the quadratic problem for all $\lambda \geq 0$ ) which begins with constraints looser than (9) and which, while retaining (8) $v^{\prime} x=0$, should alter the variables until (9) obtains. The method described here exploits this ingenious idea, differing from the proposal of [6] in keeping to a linear programming format. 


\section{THE COMPUTATION}

Here we present the computational algorithms for the minimization of $\lambda p x+1 / 2 x^{\prime} C x$ subject to $x \geq 0, A x=b$. First is given the "short form," for $\lambda$ fixed, whose convergence requires that either $\lambda=0$ or that $C$ be positive definite; next is given the "long form," solving the quadratic problem "parametrically" for all $\lambda \geq 0$, which does not need $C$ positive definite, but which involves two recursions of the "short form" type.

We shall suppose below, relying on [7], that the constraints $A x=b$ and the constraints employed below are all nondegenerate.

\section{Short Form}

Let $z^{1}, z^{2}$, and $w$ be respectively $n$-, $n$-, and $m$-component vectors. We begin with the set of relations

$$
\begin{aligned}
& A x+w=b, \\
& C x-v+A^{\prime} u+z^{1}-z^{2}=-\lambda p^{\prime}, \\
& x, v, z^{1}, z^{2}, w \geq 0,
\end{aligned}
$$

a weakening of the set (9).

Initiation. Since $b \geq 0$, an initial basis for this system can be formed from the coefficients of $z^{1}, z^{2}$, and $w$. Use the simplex method to minimize

$$
\Sigma_{i} w_{i}
$$

to zero, keeping $v$ and $u$ zero. Discard $w$ and the unused components of $z^{1}$, $z^{2}$; let the remaining $n$ components be denoted by $Z$, and their coefficients by $E$. We now have a solution of the system

$$
\begin{array}{ll}
A x & =b, \\
C x-v+A^{\prime} u+E Z & =-\lambda p^{\prime}, \\
x, v, Z \geq 0 . &
\end{array}
$$

Recursion. Given a basis and basic solution satisfying (14), (8) $v^{\prime} x=0$, and $\sum_{k=1}^{n} Z_{j}>0$, make one change of basis in the Simplex procedure for minimizing the linear form

$$
\sum_{k=1}^{n} Z_{k}
$$

under the side condition

$$
\begin{aligned}
\text { for } k=1, \ldots, n: & \text { if } x_{k} \text { is in the basis, do not admit } v_{k} ; \\
& \text { and if } v_{k} \text { is in the basis, do not admit } x_{k} .
\end{aligned}
$$

Termination. If the form (15) is positive, repeat the recursive step. The form will vanish in at most $\left(\begin{array}{c}3 n \\ n\end{array}\right)$ iterations, yielding $Z=0$. The $x$-part of 
the terminal basic solution is a solution of the quadratic programming problem for $\lambda$.

\section{Long Form}

Initiation. Having performed the short form computation for $\lambda=0$, add $p^{\prime}$ to the short form data, obtaining the system

$$
\begin{array}{ll}
A x & =b, \\
C x-v+A^{\prime} u+\mu p^{\prime}+E Z & =0,
\end{array}
$$

and an initial solution having $\mu=0, Z=0$, and $v^{\prime} x=0$.

Recursion. Given a basis and basic solution satisfying (17), (18), $v^{\prime} x=0$, and having $Z=0$, make one change of basis (if possible) in the Simplex procedure for minimizing the linear form

$$
-\mu
$$

under the side condition (16) and

$$
\text { do not allow any } Z_{j} \text { in the basis. }
$$

Termination. If it is not possible to make the basis change of the recursion, then $\mu=0, F(\lambda)=-\infty$ for all $\lambda>0$, and a set of feasible $x$ can be found (in Section 5) on which $f(\lambda, x) \rightarrow-\infty$ for $\lambda>0$.

Otherwise the recursion will yield the finite sequence of values $0=\mu^{0}<$ $\mu^{1}<\ldots<\mu^{K}$ and the $x$-parts $x^{0}, x^{1}, \ldots, x^{K}$ of their associated basic solutions, terminating in at most $\left(\begin{array}{l}2 n \\ n\end{array}\right)$ iterations with the vector $x^{\infty}$, such that: for $\mu^{k} \leq \lambda \leq \mu^{k+1}$ the quadratic problem for $\lambda$ is solved by

$$
x=\frac{\mu^{k+1}-\lambda}{\mu^{k+1}-\mu^{k}} x^{k}+\frac{\lambda-\mu^{k}}{\mu^{k+1}-\mu^{k}} x^{k+1},
$$

and for $\lambda \geq \mu^{K}$ it is solved by

$$
x=x^{K}+\left(\lambda-\mu^{K}\right) x^{\infty} .
$$

Note. E. M. L. Beale recently communicated an elegant modification of the "short form" procedure above which permits its use in the case that the quadratic form is only positive semidefinite instead of definite. It consists essentially in calculating the effect of a "virtual perturbation" of $C$ which involves replacing $C j j$ by $C j j+\gamma^{j}, j=1, \ldots, n$, for arbitrarily small $\delta$, so that the algorithm can operate as if a positive definite form were employed.

\section{EXAMPLE}

As an example for calculating with both the short form and the long form, we shall solve the problem 
Min $1 / 2\left(x_{1}^{2}+x_{2}{ }^{2}+x_{3}{ }^{2}\right)+\lambda\left(x_{1}-2 x_{3}\right)$

subject to $x_{1}, x_{2}, x_{3} \geq 0, x_{1}-x_{2}+x_{3}=1$.

The objective function can be written

$$
f(\lambda, x)=1 / 2\left[\left(x_{1}+\lambda\right)^{2}+x_{2}{ }^{2}+\left(x_{3}-2 \lambda\right)^{2}\right]-5 / 2 \lambda^{2},
$$

and thus for any $\lambda$ the solution $x$ will be that point of the constraint set closest to the point $(-\lambda, 0,2 \lambda)$. This is illustrated in Figure 1 for $\lambda=1$, and in Figure 2 for general $\lambda \geq 0$.

For this problem

$$
A=\left[\begin{array}{ccc}
1-1 & 1
\end{array}\right]
$$$$
C=\left[\begin{array}{lll}
1 & 0 & 0 \\
0 & 1 & 0 \\
0 & 0 & 1
\end{array}\right],
$$$$
p=\left[\begin{array}{lll}
1 & 0 & -2
\end{array}\right] \text {. }
$$

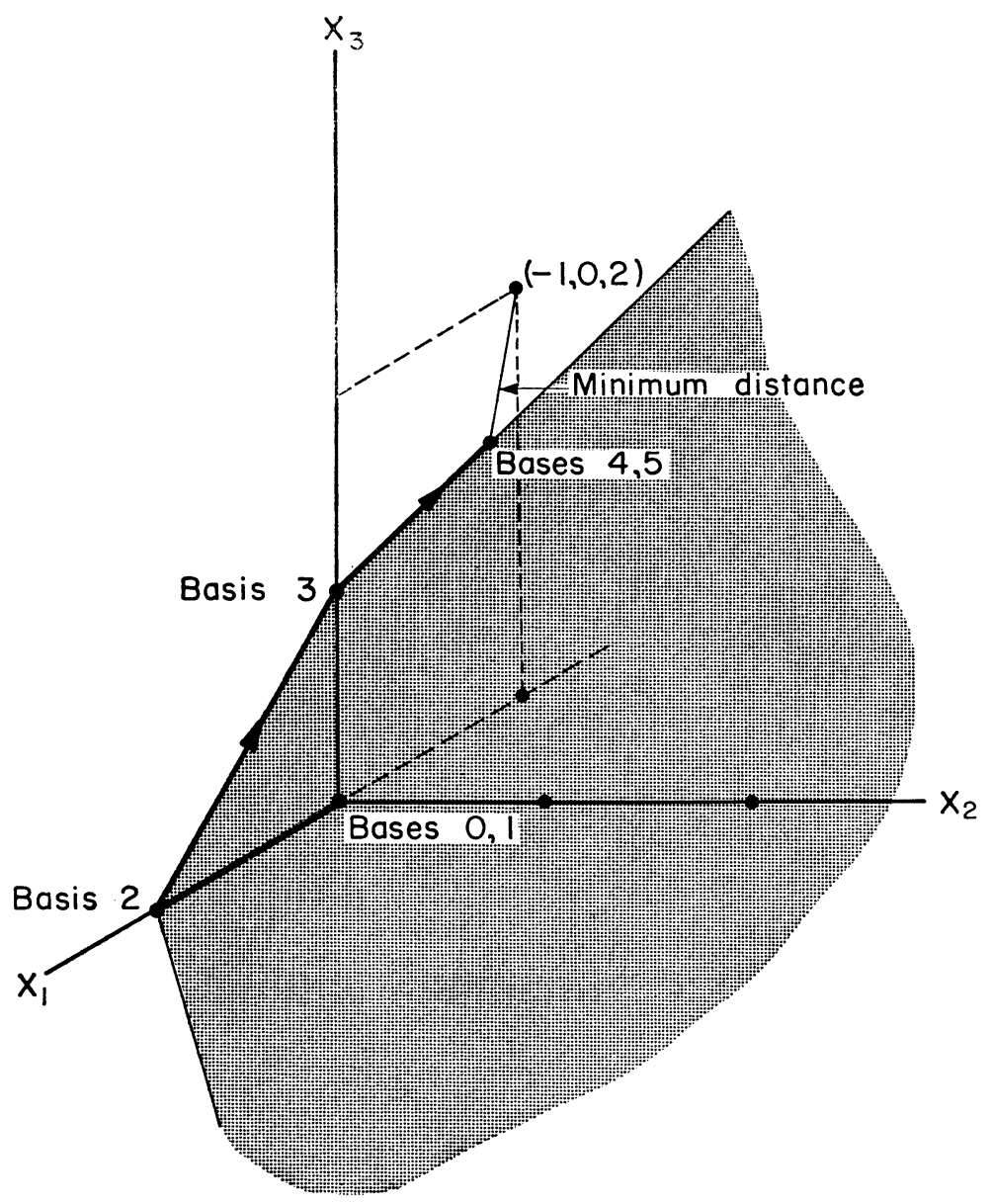

FIGURE 1-Short form. 


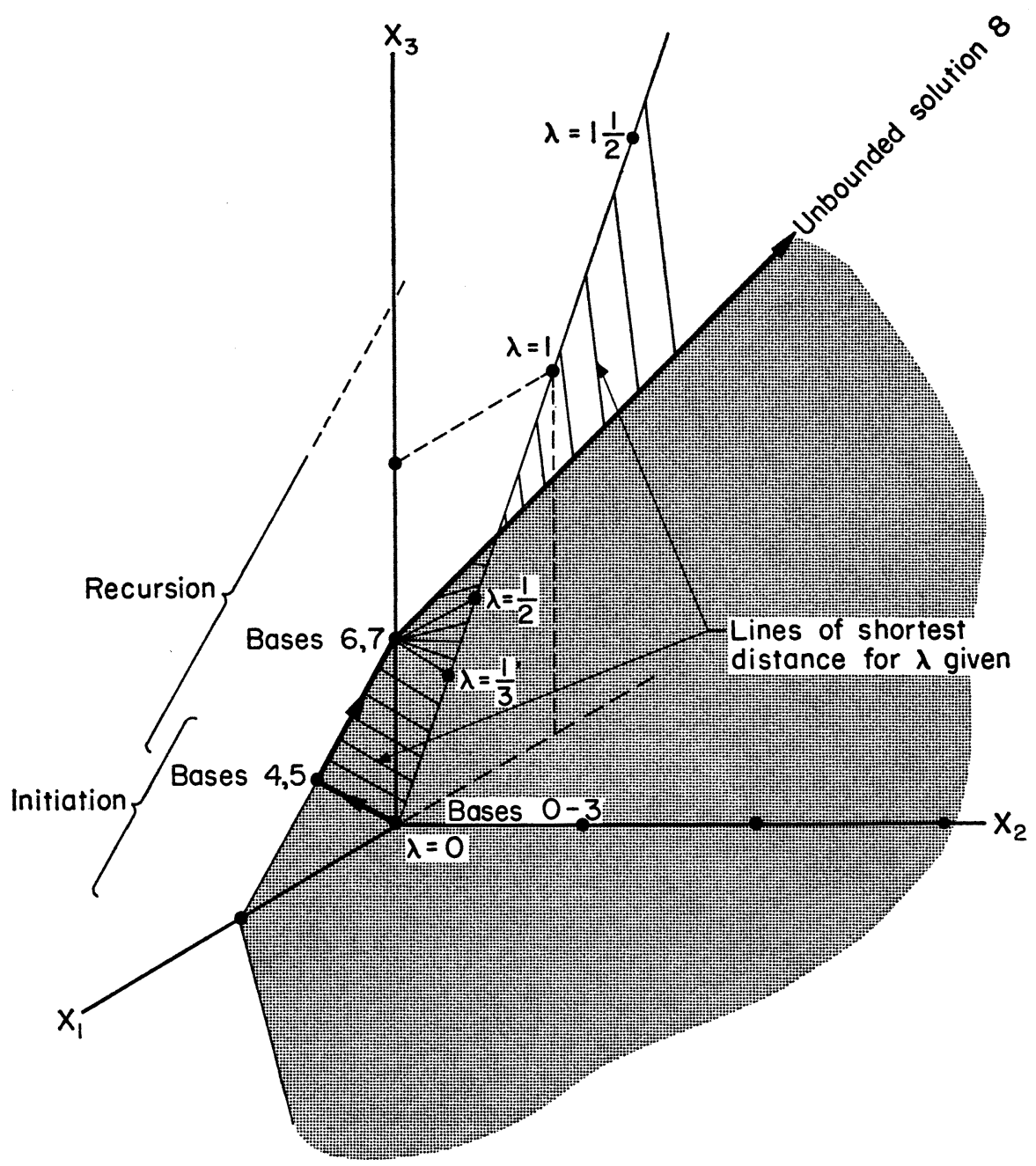

Figure 2-Long form.

Since $C$ is positive definite, the short form will solve the problem for any $\lambda$. Taking $\lambda=1$, formulas $(10,11)$ give the initial array for the short form

\begin{tabular}{|c|c|c|c|c|c|c|c|c|c|c|c|c|c|c|}
\hline$x_{1}$ & $x_{2}$ & $x_{3}$ & $v_{1}$ & $v_{2}$ & $v_{3}$ & $u$ & $z_{1}^{1}$ & & $z_{3}^{1}$ & $z_{1}^{2}$ & $z_{2}^{2}$ & $z_{3}^{2}$ & $w$ & \\
\hline & & 1 & 0 & 0 & 0 & 0 & 0 & 0 & 0 & 0 & 0 & 0 & 1 & $=1$ \\
\hline 1 & 0 & 0 & -1 & 0 & 0 & 1 & 1 & 0 & 0 & -1 & 0 & 0 & 0 & $=-1$ \\
\hline 0 & 1 & 0 & 0 & -1 & 0 & -1 & 0 & 1 & 0 & & -1 & 0 & 0 & $=0$ \\
\hline 0 & 0 & 1 & 0 & & -1 & 1 & 0 & 0 & 1 & 0 & & -1 & 0 & $=2$ \\
\hline
\end{tabular}


Although there is considerable degeneracy in the problem, the minimization of $\Sigma Z_{k}$ proceeds without a hitch. Below are given the values of the variables in the successive steps (only the values of the basic variables are given). The variable $u$ is introduced first, since it will be in every basis. Since it is unrestricted, it might have been eliminated from the system, but we haveleft it in.

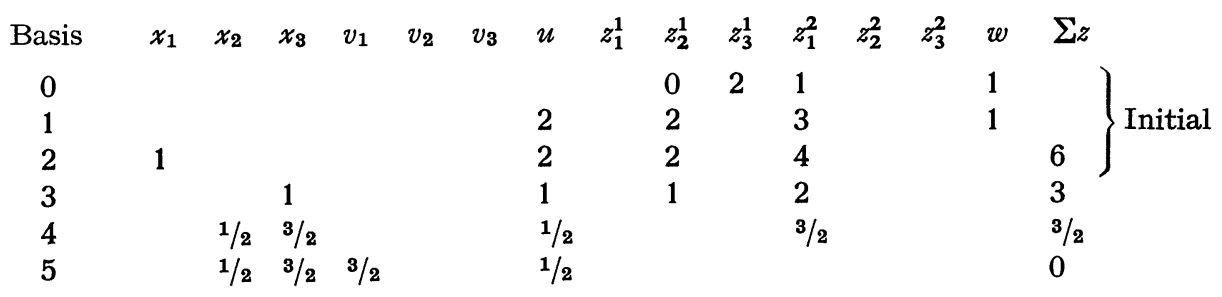

The path traced out by $x$-from $(1,0,0)$ to $(0,0,1)$ to $(0,1 / 2,3 / 2)$-is sketched in Figure 1.

For the long form, the initial array for the problem is

\begin{tabular}{|c|c|c|c|c|c|c|c|c|c|c|c|c|c|c|c|}
\hline$x_{1}$ & $x_{2}$ & $x_{3}$ & $v_{1}$ & $v_{2}$ & $v_{3}$ & $u$ & $\mu$ & $z_{1}^{1}$ & $z_{2}^{1}$ & $z_{3}^{1}$ & $z_{1}^{2}$ & & $z_{3}^{2}$ & $w$ & \\
\hline & & 1 & 0 & 0 & 0 & 0 & 0 & 0 & 0 & 0 & 0 & 0 & 0 & 1 & $=1$ \\
\hline 1 & 0 & 0 & -1 & 0 & 0 & 1 & 1 & 1 & 0 & 0 & -1 & 0 & 0 & 0 & $=0$ \\
\hline 0 & 1 & 0 & 0 & & 0 & -1 & 0 & 0 & 1 & 0 & & -1 & 0 & 0 & $=0$ \\
\hline 0 & 0 & 1 & 0 & & -1 & 1 & -2 & 0 & 0 & 1 & 0 & & -1 & 0 & $=0$ \\
\hline
\end{tabular}

The sequences of values are:

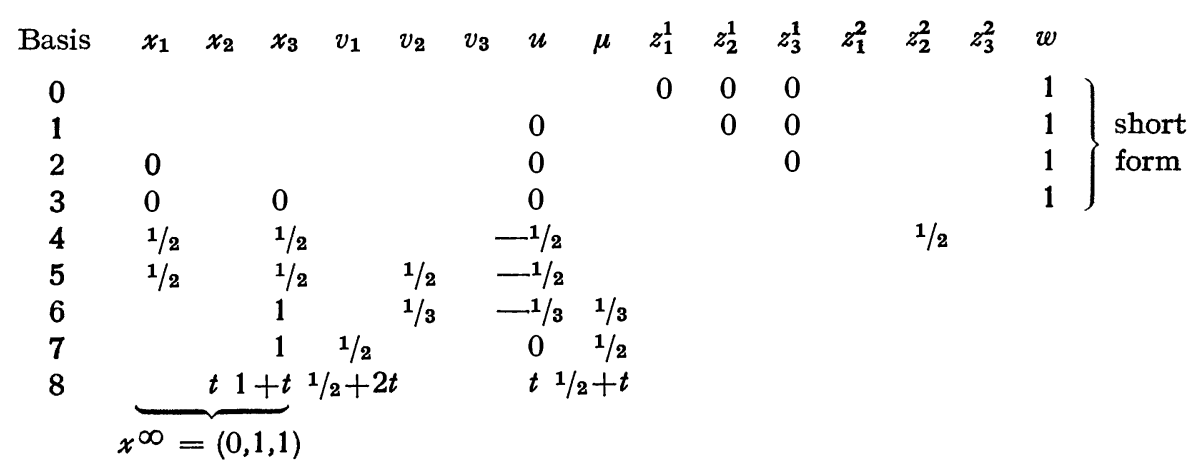

The $x$-parts of these solutions are traced out in Figure 2. 
For example, the solution of the problem for $\lambda=1 / 4$ is given by (21) as an interpolation between the Basis 5 and Basis 6 solutions as follows:

$$
x=\frac{1 / 4-1 / 3}{0-1 / 3} x^{2}+\frac{0-1 / 4}{0-1 / 3} x^{3}=1 / 4 x^{2}+3 / 4 x^{3}=(1 / 8,0,7 / 8) .
$$

The solution here for $\lambda=1$ is given directly from Basis 8 (cf. formula 22) for $t=1 / 2$ as $(0,1 / 2,3 / 2)$, the same answer the short form gave.

\section{PROOFS}

The burden of this Section is to prove the statements regarding the two terminations of the recursions of Section 3.

The initiation of the process $(v=0)$ and the side condition $(16)$ on the choice of $x$ 's and $v$ 's entering the basis have been designed to ensure that at each stage of both recursions we have $v^{\prime} x=0$. It remains to be seen what occurs when, in either recursion, it is not possible to continue the indicated minimization under the side conditions. The theorem below gives what is needed for an analysis of these conditions.

Theorem 3. Let $A, b, C$ be as in Section 1 ; let the matrix $Q$ be $n$ by $n^{\prime}, q$ be 1 by $n^{\prime}$, and $g$ be $n$ by 1 . Let $x \geq 0, v \geq 0$ such that $v^{\prime} x=0$ be given. Denote by $x_{x}$ those components of $x$ which are positive, and by $v_{x}$ the corresponding components of $v\left(\right.$ note $\left.v_{x}=0\right)$; denote by $v_{v}$ the positive components of $v$, and by $x_{v}$ the corresponding components of $x$ (note $\left.x_{v}=0\right)$.

If the linear form

is minimal under the linear constraints

$$
\begin{aligned}
& v_{x}=0, \\
& x_{v}=0 ;
\end{aligned}
$$

and

$$
\begin{aligned}
& A x \\
& C x-I v+A^{\prime} u+Q w \|=b, \\
& =g,
\end{aligned}
$$

then there exists an $r$ such that $r C=0, A r^{\prime}=0$, and $q w=r g$.

Proof. (Note that (24) is precisely the linear expression of the basis restriction side condition (18).) The proof depends upon the detailed structure of the quantities $x, v, u$, w which yield the minimum of $q w$. We have already distinguished in the vectors $x, v$ the corresponding parts $x_{x}>0, v_{x}=0$, and the corresponding parts $x_{v}=0, v_{v}>0$. There remain the corresponding parts $x_{\delta}, v_{\delta}$, which, although not positive, are not required to vanish by the constraints. In (26), below, the matrix for the constraints (25) is partitioned 
in accordance with this partioning of $x$ and $v$, first vertically, then horizontally in the naturally corresponding manner (such that -I partitions into diagonal matrices).

\begin{tabular}{|c|c|c|c|c|c|c|c|c|c|}
\hline & $x_{x}>0$ & $x_{\delta} \geqslant 0$ & $x_{v}=0$ & $v_{x}=0$ & $v_{\delta} \geqslant 0$ & $v_{v}>0$ & $u$ & $w \geqslant 0$ & \\
\hline$s$ & $A_{x}$ & $A_{\delta}$ & $A_{v}$ & 0 & 0 & 0 & 0 & 0 & $=b$ \\
\hline$v_{x}$ & $C_{x x}$ & $C_{x \delta}^{\prime}$ & $C_{x v}^{\prime}$ & $-I_{x}$ & 0 & 0 & $A_{x}^{\prime}$ & \multirow{3}{*}{$Q$} & $=g_{x}$ \\
\hline$v_{\delta}$ & $C_{x_{\delta}}$ & $C_{\delta \delta}$ & $C_{\delta v}^{\prime}$ & 0 & $-I_{\delta}$ & 0 & $A_{\delta}^{\prime}$ & & $=g_{\delta}$ \\
\hline \multirow[t]{3}{*}{$v_{v}$} & $C_{x v}$ & $C_{\delta v}$ & $C_{v v}$ & 0 & 0 & $-I_{v}$ & $A_{v}^{\prime}$ & & $=g_{v}$ \\
\hline & $\|$ & $\Lambda$ & & & $\mathbb{\Lambda}$ & $\|$ & $\|$ & $\wedge$ & \\
\hline & 0 & 0 & 0 & 0 & 0 & 0 & 0 & $q$ & \\
\hline
\end{tabular}

According to the hypothesis of the theorem, the values of the variables above this array minimize the linear form whose coefficients appear below the array. The columns corresponding to $x_{v}=0, v_{x}=0$ can be disregarded, since we insist that these variables vanish.

At the left of the array stand the variables of the linear programming problem dual to ours [8]. The coefficients of the linear form for the dual problem are those on the right of the array; the constraints for the dual problem, read vertically from the matrix, have their constant coefficients on the bottom of the array. The dual variables are unrestricted in sign since they are connected with equation constraints. The existence of these variables, satisfying the relations indicated below the matrix, is the consequence of the duality theorem for linear programming [8], as is the equality of the two objective functions. (Note particularly that where a variable turns out to be non-zero, as in $x_{x}$, or is never restricted, as in $u$, the corresponding dual relation is an equality.)

In detail, these relations are:

(a) $s A_{x}+r_{x} C_{x x}+r_{\delta} C_{x_{\delta}}+r_{v} C_{x v}=0$

(b) $s A_{\delta}+r_{x} C_{x \delta}^{\prime}+r_{\delta} C_{\delta \delta}+r_{v} C_{\delta v} \leq 0$

(c) $-r_{\delta} I_{\delta} \leq 0$

(d) $-r_{v} I_{v} \quad=0$

(e) $\quad r_{x} A_{x}^{\prime}+r_{\delta} A_{\delta}^{\prime}+r_{v} A^{\prime}{ }_{v}=0$

$\left[r_{x} \gamma_{\delta}^{\gamma} v\right] Q$

$\leq q$

and

(g)

$$
q w=s b+r g,
$$

which expresses the equality of the objectives. 
Relations (d) and (c) yield at once $r_{v}=0, r_{\delta} \geq 0$. Dropping therefore $r_{v}$, multiplying (a) on the right by $r_{x}^{\prime}$ and (b) by $r_{\delta}^{\prime}$, we have

$$
\begin{aligned}
& s A_{x} r_{x}^{r^{\prime}}+r_{x} C_{x x} \underset{x}{\gamma^{\prime}}+r_{\delta} C_{x \delta}{ }_{x}^{r^{\prime}}=0 \text {, } \\
& s A_{\delta} r_{\delta}^{\prime}+r_{x} C_{x \delta}^{\prime} r_{\delta}^{\prime}+r_{\delta} C_{\delta \delta} r_{\delta}^{\prime} \leq 0 \text {, }
\end{aligned}
$$

which are added to form

$$
s\left[A_{x} r_{x}^{r_{x}^{\prime}}+A_{\delta}^{\gamma_{\delta}^{\prime}}\right]+\left[{ }^{r}{ }_{x}^{r_{\delta}}\right]\left[\begin{array}{l}
C_{x x} C_{x \delta} \\
C_{x \delta}^{\prime} C_{\delta \delta}
\end{array}\right]\left[r_{x^{r}}{ }^{r_{\delta}}\right]^{\prime} \leq 0 .
$$

By (e), however, the first term here vanishes; and the matrix of $C$ 's in the second term, being a principal submatrix of a positive semidefinite matrix is itself positive semidefinite, so that the second term is in fact zero; whence, by Lemma 1 ,

$$
\left[\begin{array}{ll}
C_{x x} C_{x \delta} \\
C_{x \delta}^{\prime}
\end{array} C_{\delta \delta}\right]\left(r_{x} r_{\delta}\right)^{\prime}=0
$$

or

$$
\begin{aligned}
& C_{x x^{2}}{ }^{\prime}+C_{x \delta}{ }_{x \delta}^{\prime}=0, \\
& C_{x \delta}^{\prime}{ }_{x}^{\prime} r_{x}^{\prime}+C_{\delta \delta} r_{\delta}^{\prime}=0 .
\end{aligned}
$$

Equation (a) then yields just

whence we have

$$
s A_{x}=0,
$$

$$
s b=s\left[A_{x} x_{x}+A_{\delta} x_{\delta}+A_{v} x_{v}\right]=s A_{x} x_{x}=0,
$$

which, by (g), proves the theorem, letting $r=\left[\begin{array}{lll}r_{x} & r_{\delta} & r_{v}\end{array}\right]$ and noting (27e) and (28).

This theorem can be applied to the short form computation by letting

$$
Q=E, \quad q=(1, \ldots, 1), \quad \text { and } g=-\lambda p^{\prime} .
$$

When, in the course of minimizing $\sum Z_{k}$, it is not possible to reduce it under the basis restrictions, the hypothesis of Theorem 3 will be satisfied, and we will have

$$
\Sigma Z_{k}=q w=r g=-\lambda r p^{\prime},
$$

with $r C=0$. Thus, either in the case that $C$ is positive definite-when necessarily $r=0$ - or in the case that $\lambda=0$ we have $\Sigma Z_{k}=0$, so that the hypothesis of Theorem 2 is satisfied, and the terminal $x$ solves the quadratic problem.

Having reduced $Z$ to zero, we maintain this in the long form and proceed to minimize $-\lambda$. Theorem 3 is applied here by letting

$$
Q=p^{\prime}, \quad q=-1, \quad g=0 .
$$


If the long form recursion ends in a finite minimum for $-\lambda$, the hypothesis of Theorem 3 is satisfied, whence we conclude

$$
-\lambda=q w=r g=0 ;
$$

$-\lambda$ has in fact not been reduced. Two cases are thus possible: (i) no step which lowers $-\lambda$ can be taken; and (ii) $-\lambda$ can be reduced to $-\infty$.

Case (i): Here we must make use of the achievable nondegeneracy of the constraints (26) for this system, which asserts that $m+n$ of the variables in any solution are positive. Since $\lambda=0, m$ of these are in $u$, and the remaining $n$ in $x_{x}$ and $v_{v} ; x_{\delta}, v_{\delta}$ are empty. Since $A r^{\prime}=0, C r^{\prime}=0$, and, from (27f) $p r^{\prime} \leq-1$, we have that for any $t$

$$
\begin{aligned}
& A\left(x+t r^{\prime}\right)=b, \\
& C\left(x+t r^{\prime}\right)-v+A^{\prime} u=0 .
\end{aligned}
$$

It follows from nondegeneracy that $r \geq 0$; for otherwise we should have, for some $t \geq 0, x+t r^{\prime}$ satisfying (25) but vanishing in one more component than does $x$. Thus $x+t r^{\prime}$ is feasible for all $t \geq 0$, and

$$
f\left(\lambda, x+t r^{\prime}\right)=\lambda p x+1 / 2 x^{\prime} C x+\lambda p r^{\prime} t .
$$

Since $p r^{\prime} \leq-1, f\left(\lambda, x+t r^{\prime}\right) \rightarrow-\infty$ as $t \rightarrow \infty$ for any $\lambda>0$, and the desired minimum is $-\infty$.

Case (ii): The values of $\lambda$ are not bounded. Since only a finite number of bases are available, a sequence of basic solutions $\left(x^{i}, v^{i}, u^{i}, \mu^{i}\right), i=1, \ldots, g$ will be produced, and finally $\left(x^{g+1}, v^{g+1}, u^{g+1}\right)$ such that $\left(x^{g}+t x^{g+1}\right.$, $\left.v^{g}+t v^{g+1}, u^{g}+t u^{g+1}, \mu^{g}+t\right)$ is a solution for all $t \geq 0$. Due to the basis restriction (16), we will have these relations:

$$
0=v^{i} x^{i}=v^{i} x^{i+1}=v^{i+1} x^{i}=v^{i+1} x^{i+1}, \mu^{i}<\mu^{i+1} \quad(i=1, \ldots, g) .
$$

Given now that $\mu^{i} \leq \lambda \leq \mu^{i+1}$, the point

$$
x=\frac{\mu^{i+1}-\lambda}{\mu^{i+1}-\mu^{i}} x^{i}+\frac{\lambda-\mu^{i}}{\mu^{i+1}-\mu^{1}} x^{i+1},
$$

being a convex combination of $x^{i}$ and $x^{i+1}$, is feasible; and it is easy to check that, letting $v$ and $u$ be respectively the same combinations of $v^{i}, v^{i+1}$ and $u^{i}$, $u^{i+1}$, the resulting triple satisfies Theorem 3 , so that $x$ yields the desired minimum. If on the other hand $\lambda \geq \mu^{g}$, the triple $x^{g}+\left(\lambda-\mu^{g}\right) x^{g+1}$, $v^{g}+\left(\lambda-\mu^{g}\right) v^{g+1}, u^{g}+\left(\lambda-\mu^{g}\right) u^{g+1}$ satisfies Theorem 3, so that $x^{g}+$ $\left(\lambda-\mu^{g}\right) x^{g+1}$ is the answer.

\section{COMPUTATION}

Although we have presented the procedure above only for the case of constraints of the form $A x=b, x \geq 0$, relying on the fact that all types of 
linear inequality constraints may be written in this form, in practical computations there are several devices which will serve to reduce the magnitude of the problem when other types of constraints are given. We shall give these below without proofs of their effectiveness; such proofs follow closely along the line of those of Section 5 .

Let the constraints of the stated problem be

$$
\begin{array}{r}
A_{11} x_{1}+A_{12} x_{2}=b_{1}, \\
A_{21} x_{1}+A_{22} x_{2}+y_{2}=b_{2}, \\
A_{31} x_{1}+A_{32} x_{2}-y_{3}=b_{3}, \\
x_{1}, y_{2}, \quad y_{3} \geq 0 .
\end{array}
$$

(The second and third lines of (33) are the usual formulations for the constraints $\leq$ and $\geq$.) The new system of linear constraints (corresponding to (9) of Section 2) will be

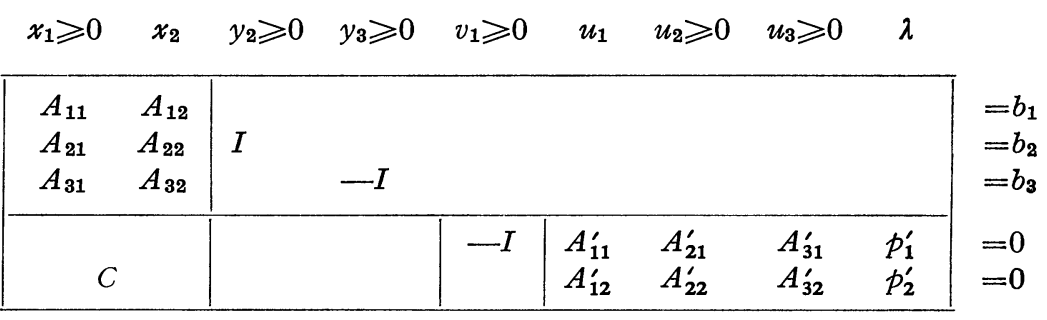

The algorithm proceeds as before, with rule (16) of Section 3 strengthened to:

If $\left(x_{1}\right)_{k}$ is in the basis, do not admit $\left(v_{1}\right)_{k}$, and vice versa;

if $\left(y_{2}\right)_{k}$ is in the basis, do not admit $\left(u_{2}\right)_{k}$, and vice versa;

if $\left(y_{3}\right)_{k}$ is in the basis, do not admit $\left(u_{3}\right)_{k}$, and vice versa.

In this formulation, it is seen that the number of equations in the stated problem is augmented only by the number of non-slack variables in the problem. A further reduction is evidently possible: since the variables $x_{2}$ and $u_{1}$ are not restricted, they could be algebraically eliminated from the system, along with an equal number of equations in which they have nonzero coefficients (this reduction could also have been performed with the $u$ of (9)). The eliminations would leave a number of equations equal to the total number of components of $x_{1}, y_{2}$, and $y_{3}$ for this generalized problem, and just the number, $n$, of components of $x$ in the simpler problem -in any case, the number of inequality constraints in the original problem. (This might seem odd if, for example, there were no inequality constraints in the stated problem; but then the operations of eliminating the unrestricted variables-all the variables-would be precisely those of solving for $x$ and $u$ in the classical Lagrange Multiplier solution.) 
While the elimination is simple to perform, we have not employed it in calculation, for the reason that it is not likely, in problems whose matrices have many zero entries, to decrease very greatly the number of nonzero entries; and it is this latter number which, to a considerable extent, determines the speed of computation in sophisticated versions of the simplex method. This must be kept in mind when estimating the relative efficiencies of procedures of this sort; in this procedure, the bulk of the data consists of the entries of $C$ and twice those of $A$. In the large problem described below, these data were only 1660 in number, although the resulting linear programming problem had 204 equations and 714 variables.

A revision of the SHARE Linear Programming code for the IBM 704 computer has been made for the solution of quadratic programming problems. This code can be used for either the short form or the long form as described above, or in an alternate version which first gets the solution to the linear problem obtained by dropping the quadratic form, and then proceeds to obtain the solutions for all $\lambda \geq 0$. The code has been used on a variety of problems, the largest of which, concerned with the allocation of a strategic material, had 90 constraints and 192 variables, 78 of which were "slacks." This problem required 359 simplex method changes-of-basis during 230 minutes for the complete long form solution.

\section{REFERENCES}

(Items [1]-[6] are concerned with computational procedures for nonlinear problems.)

[1] Barankin, E. W., And R. Dorfman: “On Quadratic Programming," University of California Publications in Statistics, 2 (1958), 285-318.

$\rightarrow$ BeAle, E. M. L.: “On Minimizing A Convex Function Subject To Linear Inequalities," Journal of the Royal Statistical Society (Ser. B) 17, 173-177 (1955).

[3] Dantzig, G. B.: Section 1 of "Recent Advances in Linear Programming," Management Science 2, 131-144 (January 1956).

[4] Frank, M. And P. Wolfe: "An Algorithm for Quadratic Programming," Naval Research Logistics Quarterly 3, 95-110 (March-June 1956).

[5] Hildreth, C.: “A Quadratic Programming Procedure," Naval Research Logistics Quarterly 4, 79-85 (March 1957).

[6] Markowitz, H.: "The Optimization of a Quadratic Function Subject to Linear Constraints," Naval Research Logistics Quarterly 3, 111-133 (March-June 1956).

[7] Dantzig, G. B., A. Orden, and P. Wolfe: "The Generalized Simplex Method for Minimizing a Linear Form under Linear Constraints," Pacific Journal of Mathematics 5, 183-195 (1955).

[8] Goldman, A. J., And A. W. Tucker: "Theory of Linear Programming," Linear Inequalities and Related Systems (H. W. Kuhn and A. W. Tucker, eds.) 53-97 (1956).

[9] Kuhn, H. W., And A. W. Tucker: "Nonlinear Programming," Proceedings of the Second Berkerley Symposium on Mathematical Statistics and Probability, 481-492 (1951). 